\title{
Macrophage Density Predicts Facial Nerve Outcome and Tumor Growth after Subtotal Resection of Vestibular Schwannoma
}

\author{
Christopher S. Graffeo ${ }^{1}$ Avital Perry ${ }^{1}$ Aditya Raghunathan ${ }^{2}$ Trynda N. Kroneman ${ }^{2}$ Mark Jentoft ${ }^{2}$ \\ Colin L. Driscoll ${ }^{1,3}$ Brian A. Neff ${ }^{1,3}$ Matthew L. Carlson ${ }^{1,3}$ Jeffrey Jacob ${ }^{4}$ Michael J. Link $k^{1,3}$ \\ Jamie J. Van Gompel 1,3
}

${ }^{1}$ Department of Neurologic Surgery, Mayo Clinic, Rochester, Minnesota, United States

2 Department of Laboratory Medicine \& Pathology, Mayo Clinic, Rochester, Minnesota, United States

${ }^{3}$ Department of Otolaryngology-Head and Neck Surgery, Mayo Clinic, Rochester, Minnesota, United States

${ }^{4}$ Departments of Neurosurgery, Michigan Head \& Spine Institute,

Royal Oak, Michigan, United States

J Neurol Surg B 2018;79:482-488.
Address for correspondence Jamie J. Van Gompel, MD, Department of Neurologic Surgery, Mayo Clinic, 200 First St SW, Rochester, MN 55905, United States (e-mail: VanGompel.Jamie@Mayo.edu).

\section{Abstract \\ Keywords \\ - vestibular schwannoma \\ - subtotal resection \\ - tumor recurrence \\ - tumor progression \\ - macrophages \\ - macrophage density}

Introduction Vestibular schwannoma (VS) behavior following subtotal resection (STR) is highly variable. Overall progression rates have been reported as high as $44 \%$, and optimal treatment is controversial. Correspondingly, identification of a reliable clinical or pathologic marker associated with progression after STR would help guide decision-making.

Methods A prospectively maintained institutional VS registry from 1999 to 2014 was retrospectively reviewed for sporadic VS patients who underwent primary STR without preceding stereotactic radiosurgery (SRS) by a single neurosurgery-neurotology team. Primary endpoints included tumor progression and postoperative facial nerve function. Pathologic specimens were stained for Ki67, CD68, S100, and SOX10 and were quantitated by digital imaging analysis. Macrophage density was defined as the ratio of $\mathrm{CD}_{68}{ }^{+}$macrophages to $\mathrm{S} 100^{+}$macrophages and Schwannian tumor cells. Clinical outcomes were correlated with pathologic markers.

Results Forty-six patients met the study inclusion criteria. Thirteen (28\%) progressed during a mean 57 months of follow-up (range 15-149). Favorable postoperative facial nerve function (House-Brackmann I-II) was achieved in $37(80 \%)$. CD68 ${ }^{+}$cells were present at significantly higher concentrations in tumors that progressed $(p=0.03)$. Higher macrophage density was significantly associated with both tumor progression $(p=0.02)$ and unfavorable facial nerve function $(p=0.02)$. Ki67 percent positivity was not significantly associated with either primary endpoint $(p=0.83 ; p=0.58)$.

Conclusions Macrophage density may provide an important marker for individuals at the highest risk for progression of VS after STR, potentially prompting closer surveillance or consideration for upfront SRS following STR. This finding supports preceding conclusions that an intratumoral macrophage-predominant inflammatory response may be a marker for tumor growth and a potential therapeutic target. received

February 10, 2017

accepted after revision

January 2, 2018

published online

February 7, 2018 (c) 2018 Georg Thieme Verlag KG

Stuttgart · New York
DOI https://doi.org/

$10.1055 / \mathrm{s}-0038-1627474$. ISSN 2193-6331. 


\section{Introduction}

Vestibular schwannoma (VS) is a benign neoplasm arising from the Schwann cells investing the vestibular branches of cranial nerve VIII with myelin. When surgical resection is performed, extent-of-resection is the most important predictor of tumor control, as recurrence after gross total resection (GTR) has been reported in 0.05 to $9.2 \%$ of surgical series, whereas subtotal resection (STR) is followed by progression of residual tumor in up to $44 \%{ }^{1-9}$ Optimal management after STR is contentiously debated, with various authors advocating for proactive stereotactic radiosurgery (SRS) of residual tumor, versus close observation followed by treatment with SRS or repeat microsurgery once tumor progression has declared itself. ${ }^{10,11}$ Still others advocate for planned STR followed by proactive SRS, to theoretically optimize postoperative facial nerve function. ${ }^{12-14}$ Consequently, a pathologic marker predictive of tumor aggressiveness may greatly help inform clinical decision making regarding the role of proactive treatment of a tumor remnant after STR, and imaging surveillance intervals after GTR.

As with many other solid tumors, VS is characterized by intra-tumor inflammatory infiltrates, and the VS immune microenvironment is an area of active study with respect to both understanding of VS biology and the development of targeted therapies. ${ }^{15} \mathrm{~A}$ major area of active research and debate is the nature of pro-tumoral and anti-tumoral activity that the immune system carries out within a broad range of solid neoplasms, including VS. ${ }^{16-20}$ Macrophages-in particular, those of tumor-promoting M2 lineage-have been previously associated with VS growth in general and are noted to be present in higher concentration among those tumors with more robust angiogenesis and marked preoperative growth. ${ }^{18,21}$ However, the role of inflammation in predicting postoperative tumor and facial nerve behavior has not been clearly established-particularly in the setting of STR. Correspondingly, we sought to study the potential relationship between intratumoral macrophages and local control after STR in a large, single-institution series of VS.

\section{Methods}

\section{Clinical and Radiographic Review}

A prospectively maintained single-institution VS database was retrospectively queried for all individuals from 1999 to 2014 who underwent STR by a neurosurgery-neurotology team. Patients with a history of prior microsurgery or SRS for treatment of ipsilateral VS were excluded, as were patients with NF2. Clinical endpoints captured included age, sex, preand postoperative American Academy of OtolaryngologyHead and Neck Surgery (AAO-HNS) hearing grade, surgical approach, surgeon's impression extent-of-resection, postoperative House-Brackmann grade facial nerve function, cerebrospinal fluid (CSF) leak, and time to last clinical follow-up. ${ }^{22}$ Unfavorable facial nerve function was defined as House-Brackmann III-VI. ${ }^{23}$ All patients had intact facial nerve function at baseline, and outcomes are reported at time of last follow-up, which was at least 1 year for all included individuals. Additional treatments were documented, including proactive SRS of residual tumor, SRS of progressive tumor, or repeat microsurgery of progressive tumor. Preoperative magnetic resonance imagings (MRIs) were reviewed to document preoperative tumor size (measured using the point of largest apparent diameter in the axial plane, excluding the IAC; calculated using an average of three measurements), radiographic confirmation of STR (defined as nodular enhancement within the cerebellopontine angle (CPA) or internal auditory canal (IAC) on first postoperative MRI usually performed 3 months postoperatively), and radiographic evidence of tumor progression (defined as $\geq 2 \mathrm{~mm}$ increase in nodular enhancement following radiographically confirmed STR). Preoperative growth rates were calculated for patients with at least two preoperative MRIs and at least 6 months of preoperative follow-up ( - Table 1; $n=15$; median 24 months, range 6-48).

\section{Pathologic Review and Digital Image Analysis}

All available slides from specimens were centrally reviewed by a study neuropathologist (Aditya Raghunathan) who was blinded to the clinical outcomes, and the most representative section was selected. Consecutive $4-\mu \mathrm{m}$ sections were prepared from the formalin-fixed paraffin-embedded tissue block and were stained using standard techniques for hematoxylin and eosin (H\&E) and by immunohistochemistry (IHC) for Ki67 (MIB-1 clone; 1:20), CD68 (KP1 clone; 1:1500), S100 (polyclonal; 1:4000), and SOX10 (BC34 clone; 1:200). Whole slide scanning of the H\&E and IHC stained slides was performed at 400x magnification on the Aperio ScanScope AT Turbo brightfield instrument (Leica Biosystems) at a resolution of 0.25 microns per pixel. The images were 24-bit contiguous standard pyramid tiled TIFFs compressed via JPEG2000 with a quality setting of 70 . The selected regions of interest (ROI) were negative for hemorrhage and artifactual tissue distortion and were largely comprised of representative fields on H\&E and Ki67 IHC (-Fig. 1A-C). The ROI was then digitally transposed onto the scanned sections of the other IHC preparations. For digital image analysis, the Aperio ImageScope Software (Leica Biosystems) was utilized. Nuclear and cytoplasmic algorithms were optimized for each $\mathrm{IHC}$, and the total number of positive staining cells, percent of positive cells of the total cells, and total area of analysis were calculated in the ROIs (- Fig. 1C). Standardized positive staining values were calculated for statistical analysis by dividing the number of positive nuclei by the area of analysis. Macrophage density was calculated as the ratio of $\mathrm{CD} 68^{+}$ nuclei to $\mathrm{S} 100^{+}$nuclei per sampled region.

\section{Statistical Analysis}

Statistical tests included Student's $t$-test or exact Wilcoxon rank-sum for continuous data and Fisher's exact or Chisquare test for categorical data, as appropriate. Statistical analyses were performed using SAS 9.4 and JMP 10.0.0 (SAS Institute Inc., Cary, North Carolina, United States, 19892012). All tests were two-sided, and $p$-values $<0.05$ were considered statistically significant. 
Table 1 Study cohort overview

\begin{tabular}{|c|c|c|c|}
\hline & $\begin{array}{l}\text { Stable disease } \\
(n=33)\end{array}$ & $\begin{array}{l}\text { Tumor progression } \\
(n=13)\end{array}$ & $p$-Value \\
\hline \multicolumn{4}{|l|}{ Demographics and baseline characteristics } \\
\hline Female sex & $20(61 \%)$ & $7(54 \%)$ & 0.68 \\
\hline Age at diagnosis (y; median (range)) & $57(25-83)$ & $57(19-86)$ & 0.98 \\
\hline Class A-B hearing (preoperative assessment) & $8(24 \%)$ & $5(38 \%)$ & 0.47 \\
\hline Total postoperative follow-up (mo; median (range)) & $39(15-149)$ & $45(29-135)$ & 0.17 \\
\hline \multicolumn{4}{|l|}{ Radiographic features } \\
\hline Preoperative tumor size (cm; median (range)) & $3.5(1.8-5.2)$ & $2.9(1.8-5.0)$ & 0.41 \\
\hline Macrocystic tumor architecture & $8(24 \%)$ & $3(23 \%)$ & 0.93 \\
\hline Preoperative growth rate (mm/y; median (range)) & $6(1.8-9)^{\mathrm{a}}$ & $4.5(2.5-5.5)^{b}$ & 0.43 \\
\hline Postoperative residual volume $\left(\mathrm{mm}^{3} ;\right.$ median (range)) & $458(90-2100)$ & $304(91-2440)$ & 0.72 \\
\hline \multicolumn{4}{|l|}{ Surgical approach and postoperative outcomes } \\
\hline Restrosigmoid craniotomy & $24(73 \%)$ & $7(54 \%)$ & 0.22 \\
\hline Translabyrinthine craniotomy & $9(27 \%)$ & $6(46 \%)$ & 0.22 \\
\hline Class A-B hearing (postoperative assessment) & $0(0 \%)$ & $0(0 \%)$ & 1.0 \\
\hline Unfavorable facial nerve function (HB III-VI) & $4(12 \%)$ & $5(38 \%)$ & 0.04 \\
\hline Cerebrospinal fluid leak & $4(12 \%)$ & $1(8 \%)$ & 0.19 \\
\hline \multicolumn{4}{|l|}{ Additional treatments } \\
\hline Proactive stereotactic radiosurgery of residual tumor & $4(12 \%)$ & $3(23 \%)$ & 0.35 \\
\hline Stereotactic radiosurgery of progressive tumor & - & $6(46 \%)$ & - \\
\hline Repeat microsurgery of progressive tumor & - & $1(8 \%)$ & - \\
\hline
\end{tabular}

Abbreviations: HB, House-Brackmann; mo, month; y, year.

Boldface type indicates statistical significance at $\alpha=0.05$.

${ }^{a} n=11$.

${ }^{\mathrm{b}} n=4$.

\section{Results}

Forty-six patients met clinical and radiographic study criteria and were included in the analysis. Radiographic review confirmed 13 instances of tumor progression after STR; the remaining 33 had stable disease throughout the study period. Median clinical follow-up was 41 months (range 15-149 months). Patient demographics, baseline characteristics, radiographic features, surgical approaches, CSF leak, and rate of proactive SRS to the residual tumor were not significantly different between the groups ( - Table 1 ). Unfavorable facial nerve function was more common among individuals with tumor progression (12\% versus $38 \%$, $p=0.04)$. Median postoperative residual tumor volumes were not significantly different between the groups (458 $\mathrm{mm}^{3}$ versus $304 \mathrm{~mm}^{3}, p=0.72$ ).

Pathologic analysis noted significantly higher CD68 staining among tumors that progressed, but not among tumors with unfavorable facial nerve outcomes; CD68 percent positivity was significantly higher among both tumors that progressed and tumors that had unfavorable facial nerve outcomes (-Tables 2 and 3; -Fig. 2A). Ki67 index and positive staining for S100 and SOX10 were not significantly different between the groups, with respect to tumor control or facial nerve function. Higher macrophage density was significantly associated with both tumor progression and unfavorable facial nerve function (-Fig. 2B).

\section{Discussion}

Tumor progression may occur following STR and require further treatment with added cost and risk of additional patient morbidity. Although residual tumor growth is common, it affects fewer than half of patients after STR, and no clear biomarkers reliably predict progression. Correspondingly, critical decision-making-including the timing of, or indications for, postoperative SRS or additional surgery-remains a point of clinical controversy and is incompletely understood. In the present study, we sought to elucidate the possible role of macrophages as key players in the VS immune milieu, to assess their utility as a potential marker of outcomes after STR. ${ }^{18,21}$ Preliminarily, we observed for the first time that higher levels of macrophage-predominant inflammation are associated with progression of residual tumor. More interestingly still, we noted that when the proliferative balance within the tumor microenvironment is shifted toward macrophages and away from Schwannian tumor cells, both tumor 
A

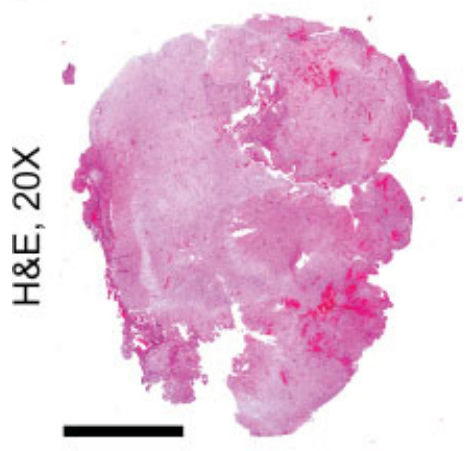

C
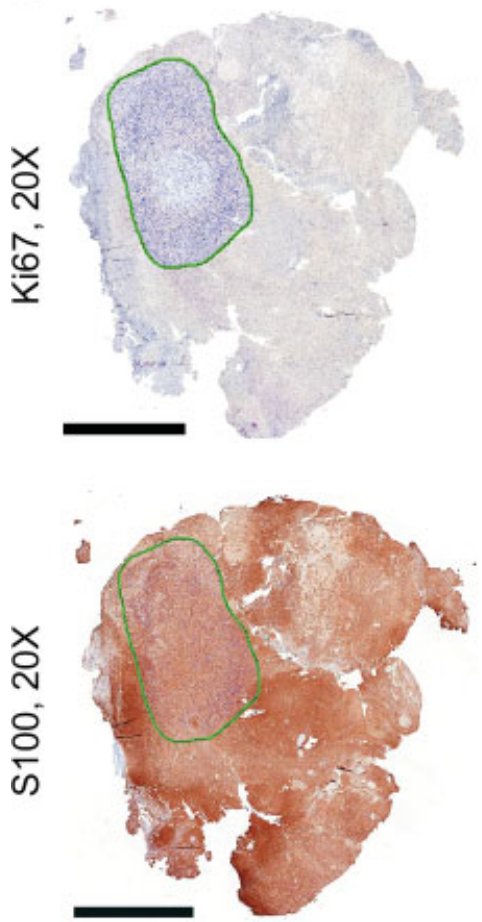

B
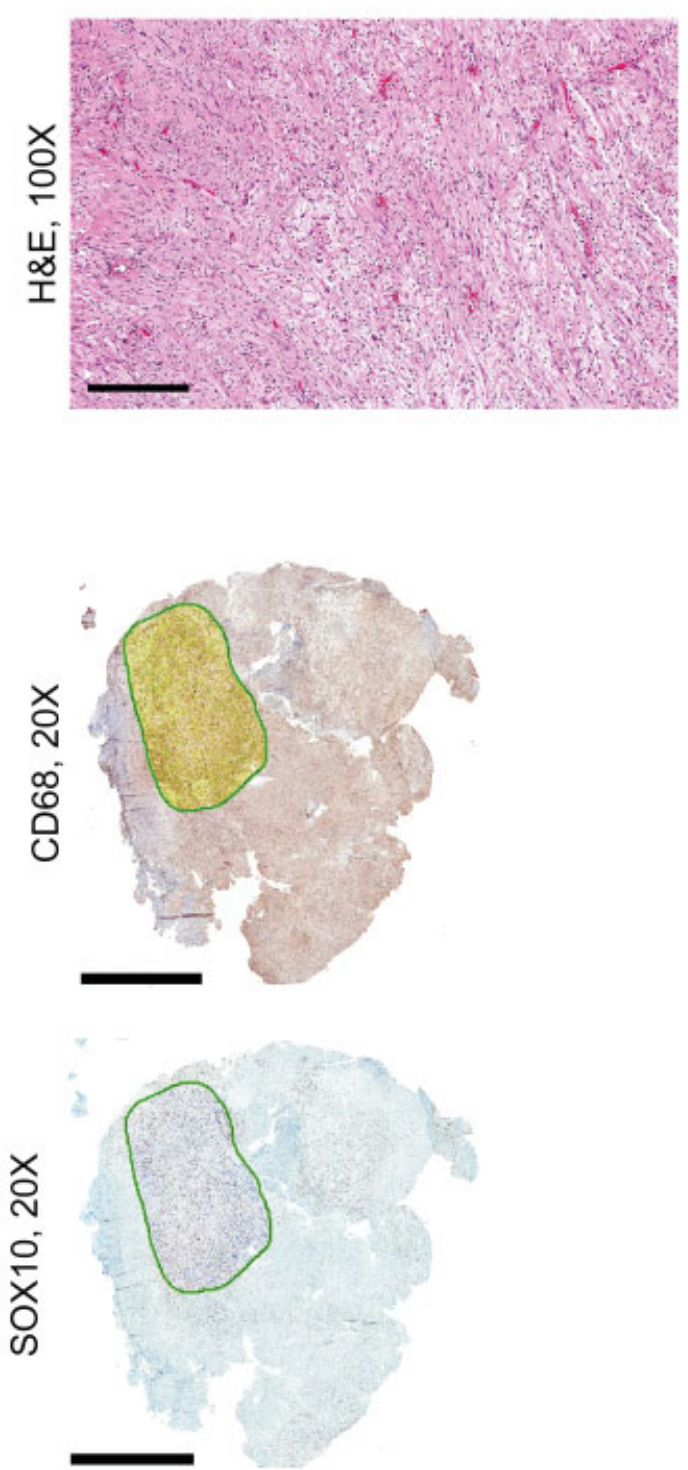

Fig. 1 Low- (A, scale bar $3 \mathrm{~mm})$ and high-powered (B, scale bar $300 \mu \mathrm{m})$ photomicrographs of a representative tumor section stained using H\&E demonstrate prototypical vestibular schwannoma features including spindle-like tumor cells with tapered nuclei, Antoni B pattern changes against a myxoid stroma, and diffuse nonspecific inflammatory cell infiltration. Adjacent sections were stained via immunohistochemistry for Ki67, CD68, S100, and SOX10, with regions-of-interest selected for quantitative analysis outlined in green (C, scale bars $3 \mathrm{~mm}$ ). H\&E, hematoxylin and eosin.

Table 2 Pathologic analysis-tumor control

\begin{tabular}{|l|l|l|l|}
\hline & $\begin{array}{l}\text { Stable disease } \\
(\boldsymbol{n}=33)\end{array}$ & $\begin{array}{l}\text { Tumor progression } \\
(\boldsymbol{n}=13)\end{array}$ & $p$-Value \\
\hline Ki67 index (\% positive, 3+ and $2+;$ median (range)) & $1.2 \%(0.5-3.7 \%)$ & $1.4 \%(0.4-3.1 \%)$ & 0.83 \\
\hline CD68 staining (positive cells/mm $;$ median (range)) & $3083(1416-5575)$ & $3848(2958-5038)$ & 0.03 \\
\hline CD68 percent positivity (median (range)) & $35 \%(16-82 \%)$ & $51 \%(8-93 \%)$ & 0.03 \\
\hline S100 staining (positive cells/mm²; median (range)) & $1542(847-2928)$ & $1816(1194-2645)$ & 0.31 \\
\hline SOX10 staining (positive cells/mm ; median (range)) & $2605(993-4388)$ & $2864(1763-4723)$ & 0.18 \\
\hline Macrophage density (CD68/S100; median (range)) & $1.9(1.4-2.4)$ & $2.2(1.7-2.5)$ & 0.02 \\
\hline
\end{tabular}

Boldface type indicates statistical significance at $\alpha=0.05$. 
Table 3 Pathologic analysis-facial nerve function

\begin{tabular}{|l|l|l|l|}
\hline & HB I-II $(\boldsymbol{n}=37)$ & HB III-VI $(\boldsymbol{n}=\mathbf{9})$ & $p$-Value \\
\hline Ki67 index (\% positive, 3+ and $2+;$ median (range)) & $1.2 \%(0.4-3.7 \%)$ & $1.3 \%(0.6-2.0 \%)$ & 0.58 \\
\hline CD68 staining (positive cells/mm²; median (range)) & $3291(1417-4732)$ & $3784(2958-5421)$ & 0.13 \\
\hline CD68 percent positivity (median (range)) & $31 \%(16-64 \%)$ & $52 \%(8-93 \%)$ & $\mathbf{0 . 0 2}$ \\
\hline S100 staining (positive cells/mm $;$ median (range)) & $1633(847-2928)$ & $1816(1194-2738)$ & 0.55 \\
\hline SOX10 staining (positive cells/mm²; median (range)) & $2726(993-4724)$ & $2532(2155-4077)$ & 0.71 \\
\hline Macrophage density (CD68/S100; median (range)) & $\mathbf{1 . 9 6 ( 1 . 3 9 - 2 . 4 1 )}$ & $\mathbf{2 . 1 1 ( 1 . 8 6 - 2 . 4 8 )}$ & $\mathbf{0 . 0 3}$ \\
\hline
\end{tabular}

Abbreviation: HB, House-Brackmann.

Boldface type indicates statistical significance at $\alpha=0.05$.

progression and unfavorable facial nerve outcomes occur at a significantly higher rate.

The observation of elevated CD68 among tumors that progressed is a logical extension of recent findings regarding macrophage activity in VS, in particular the work of de Vries et al. In a study of 68 sporadic VS, they first identified an association between $\mathrm{CD}^{+} 8^{+}$macrophages and VS size and intratumoral angiogenesis; this was followed by an analysis comparing 10 fast growing VS to 10 slow growing VS, which more specifically examined the $\mathrm{CD}_{163^{+}} \mathrm{M} 2$ macrophage subpopulation and demonstrated their association with accelerated tumor growth, as well as increased angiogenesis. ${ }^{18,21}$ Taken together, these findings and our own results suggest that macrophages are important markers of tumor growth throughout the stages of VS natural history and that they may act to down-regulate the overall immune response, potentiating tumor growth. ${ }^{17}$

Consensus regarding the $\mathrm{Ki67}$ index as a reliable marker of proliferation has been less unanimous, particularly with regard to its predictive value in the assessment of VS growth. While Niemzyk et al observed a significant correlation between elevated Ki67 index and tumor growth, GomezBrouchet et al and others have failed to demonstrate that elevated Ki67 is associated with tumor growth and have suggested that the stain may be subject to significant sam- pling bias when used in VS analyses. ${ }^{21,24,25}$ We, as well, failed to find a correlation between Ki67 and tumor aggressiveness following STR, highlighting the need for a more reliable, sensitive, and specific marker of VS growth.

The significance of the novel association we identified between macrophage density and both tumor progression and unfavorable facial nerve function raises several interesting points. S100 positivity is observed in both macrophages and Schwann cells in the tumor, meaning that the higher ratio of CD68 to $\mathrm{S} 100$ cells indicates a relative decrease in the fraction of cells staining for S100 alone. Our preliminary interpretation is that this signals a local change in which immune cell proliferation overtakes that of tumor cells and that this macrophage-predominant process is at minimum a marker of underlying, insidious changes within the tumor microenvironment that predispose to a more aggressive phenotype.

In addition to driving tumor growth, a more active intratumor immune milieu may result in heightened inflammation at the facial nerve-tumor interface, potentially predisposing to a more difficult dissection, and therefore a higher rate of poor facial nerve outcomes. Alternatively, increased inflammation may signal a sensitized facial nerve with diminished reserve, making it vulnerable to even minor manipulation. Similar phenomena have been theorized, for
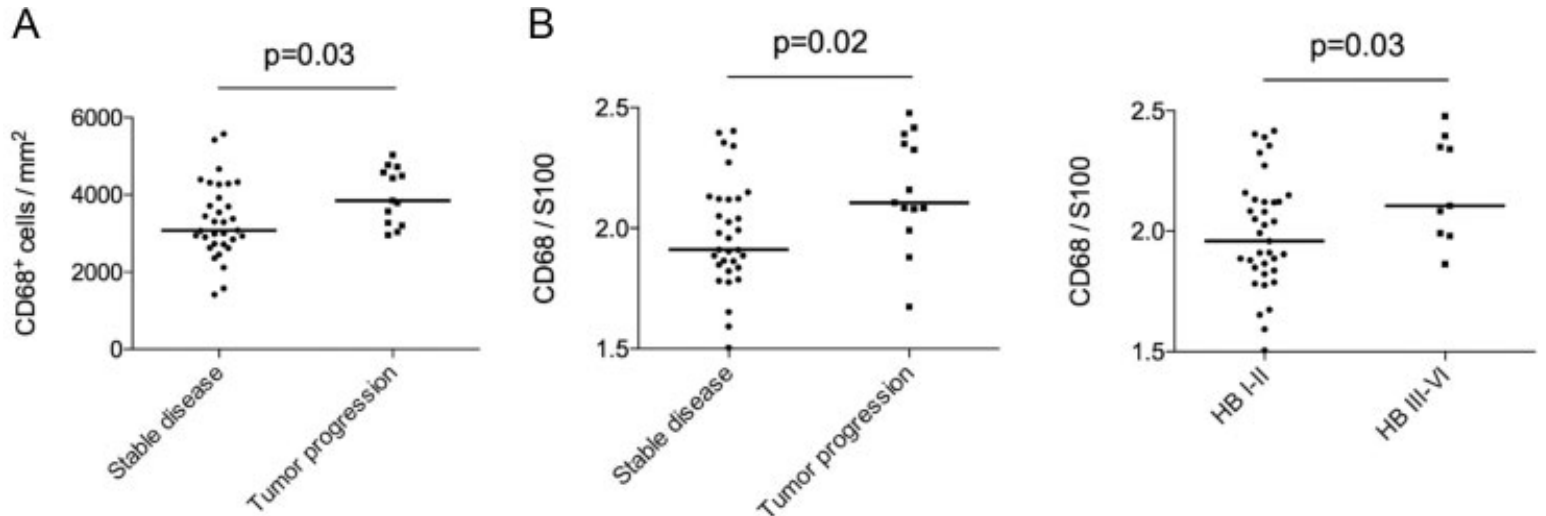

Fig. 2 Graphical representations of CD68 staining (A) and macrophage density (B) demonstrate significant differences with respect to tumor control and facial nerve function. HB, House-Brackmann. 
example, in the setting of delayed hemifacial spasm (HFS) after VS treatment with SRS. For instance, Pollock has noted that, when managed with medical agents targeted at reducing nerve irritation, such as steroids and anticonvulsants, HFS typically abates; whereas if the spasm was managed with tumor resection, the facial nerve was subject to an elevated risk of injury, likely due to scarring and inflammation at the facial nerve-tumor interface. ${ }^{26,27}$

Our parallel finding of a significant, independent association between tumor progression and unfavorable facial nerve function lends additional support to the theory that pathologic inflammation underlies both processes-particularly given that there was not a significant difference in postoperative residual tumor volumes between the groups. This is made especially interesting by the counterintuitive nature of the finding: from a clinical perspective, one might conceptualize stable disease as a marker of a more aggressive resection, and therefore a risk factor for unfavorable facial nerve function. That we observed instead the inverse relationship suggests an alternative mechanism, in which a feature of the tumor microenvironment that was present at the time of resection (heightened inflammation) is associated with both a sensitized facial nerve that was predisposed to injury and a more proliferative tumor that will be more likely to progress following STR. While we cannot prove a correlation between intra-tumor inflammation manifest as increased macrophage density and tumor aggressiveness, we feel this is an important area of future research.

The potential clinical implications of our findings are more salient in terms of postoperative management. In patients known to harbor tumor remnants with heightened inflammation, closer surveillance may be warranted, while electing to delay SRS until progression might decrease the risk of complications, such as delayed facial nerve paralysis and HFS. Conversely, if a high-efficiency assay or pathologic technique were developed to provide intraoperative data such as macrophage density, this may helpfully inform intraoperative decision making, compelling the surgeon to either not engage the facial nerve altogether and proceed with deliberate STR followed by proactive SRS in a patient who strongly desired to avoid facial weakness or proceed with aggressive resection and anticipate facial weakness, with the goal of minimizing the long-term risk of recurrence in a tumor that is likely to be more difficult to treat.

Most importantly, the emerging role of macrophages in the VS tumor microenvironment highlights an important potential avenue for targeted therapies. Although it remains to be proven that the macrophages are indeed pro-tumor actors within the VS microenvironment, our findings provide some limited support that there may be clinical utility in the on-going efforts to develop agents that shift macrophage differentiation toward the M1 antitumor phenotype, as well as more conventional inhibitors of M2 macrophage attraction and induction. ${ }^{16,28-30}$ This is particularly of interest when considered in the context of our finding that some tumor fields were comprised of up to 93\% macrophages, identifying a potentially robust target for biologic agents, which we suspect might have meaningful efficacy against tumors with at least $50 \%$ CD68 percent positivity. To this end, there already exists substantial research regarding the role of immunomodulatory agents across a wide variety of diseases, several of which we anticipate may ultimately prove relevant to the treatment of VS. ${ }^{31}$

Of particular interest is aspirin, a well-characterized pharmaceutical with potent anti-inflammatory properties involving several key pathways, most prominently cyclooxygenase-2, and nuclear factor (NF)-кB. Recent analyses studying the in vivo response to aspirin prescribed for unrelated indications in patients with sporadic VS have shown significantly decreased tumor growth in both linear and volumetric analyses, as compared with patients not taking aspirin. ${ }^{31,32}$ Parallel in vitro findings have confirmed that aspirin therapy produces significant cyclooxygenase-2-mediated cytostatic inhibition of VS cell proliferation, highlighting an important avenue for prospective study, potentially to the end of a promising nearfuture immunomodulatory intervention using a safe and well-studied medication. ${ }^{33}$ In our study, data regarding aspirin use was collected, but was ultimately excluded from the final analysis, due to significant heterogeneity in the retrospectively data extracted from our medical record. Among other factors, a meaningful and accurate analysis was prohibited by variability in patient dosing schemes and unclear or inconsistent documentation with regard to the timing of medication administration (e.g., was the medication chronically taken preoperatively, postoperatively, or both?), as well as any concomitant nonsteroidal anti-inflammatory drugs (NSAID) use. Concerns regarding patient recall bias were also weighed, given that most of the data were also retrospectively reported by the patients at the time of clinical consultation.

The results of the present study are clearly preliminary and are subject to several significant limitations. Clinical data were retrospectively reviewed, and our group sizes were relatively small. In our pathologic analysis, we studied all $\mathrm{CD}^{+} 8^{+}$macrophages, without discriminating between M1 and M2 subtypes. Furthermore, although we did find significant differences in both CD68 staining and macrophage density, the ranges of the groups did overlap, calling into question the ultimate value of this potential marker of tumor aggressiveness.

\section{Conclusion}

The tumor immune microenvironment in VS is an important area of active investigation. We feel this may ultimately reveal a marker for tumor behavior and provide a target for therapy. Tumors with higher CD68 staining were more likely to demonstrate tumor progression. Higher macrophage density was significantly associated with both tumor progression and unfavorable facial nerve function. Although qualified by the limitations detailed above, we hold that our analysis importantly advances our understanding of several interesting and provocative questions regarding pathologic inflammation in VS. 
Financial Material and Support

Internal departmental funding was utilized without commercial sponsorship or support.

\section{Conflicts of Interest}

None.

\section{Previous Presentation}

Components of this manuscript were presented at the North American Skull Base Society 2017 annual meeting

\section{References}

1 Ahmad RA, Sivalingam S, Topsakal V, Russo A, Taibah A, Sanna M. Rate of recurrent vestibular schwannoma after total removal via different surgical approaches. Ann Otol Rhinol Laryngol 2012;121 (03):156-161

2 El-Kashlan HK, Zeitoun H, Arts HA, Hoff JT, Telian SA. Recurrence of acoustic neuroma after incomplete resection. Am J Otol 2000; 21(03):389-392

3 Freeman SR, Ramsden RT, Saeed SR, et al. Revision surgery for residual or recurrent vestibular schwannoma. Otol Neurotol 2007;28(08):1076-1082

4 Hong B, Krauss JK, Bremer M, Karstens JH, Heissler HE, Nakamura M. Vestibular schwannoma microsurgery for recurrent tumors after radiation therapy or previous surgical resection. Otol Neurotol 2014;35(01):171-181

5 Jacob JT, Carlson ML, Driscoll CL, Link MJ. Volumetric analysis of tumor control following subtotal and near-total resection of vestibular schwannoma. Laryngoscope 2016;126(08):1877-1882

6 Matthies C, Samii M. Management of 1000 vestibular schwannomas (acoustic neuromas): clinical presentation. Neurosurgery 1997;40(01):1-9, discussion 9-10

7 Roberson JB Jr, Brackmann DE, Hitselberger WE. Acoustic neuroma recurrence after suboccipital resection: management with translabyrinthine resection. Am J Otol 1996;17(02):307-311

8 Samii M, Gerganov VM, Samii A. Functional outcome after complete surgical removal of giant vestibular schwannomas. J Neurosurg 2010;112(04):860-867

9 Samii M, Matthies C, Tatagiba M. Management of vestibular schwannomas (acoustic neuromas): auditory and facial nerve function after resection of 120 vestibular schwannomas in patients with neurofibromatosis 2. Neurosurgery 1997;40(04): 696-705, discussion 705-706

10 Pollock BE, Lunsford LD, Flickinger JC, Clyde BL, Kondziolka D. Vestibular schwannoma management. Part I. Failed microsurgery and the role of delayed stereotactic radiosurgery. J Neurosurg 1998;89(06):944-948

11 Samii M, Metwali H, Gerganov V. Microsurgical management of vestibular schwannoma after failed previous surgery. J Neurosurg 2016;125(05):1198-1203

12 Iwai Y, Ishibashi K, Watanabe Y, Uemura G, Yamanaka K. Functional preservation after planned partial resection followed by gamma knife radiosurgery for large vestibular schwannomas. World Neurosurg 2015;84(02):292-300

13 Monfared A, Corrales E, Theodosopoulos P, et al. Facial nerve outcome and tumor control rate as a function of degree of resection in treatment of large acoustic neuromas: preliminary report of the Acoustic Neuroma Subtotal Resection Study (ANSRS). Neurosurgery 2016;79(02):194-203

14 van de Langenberg $R$, Hanssens PE, van Overbeeke JJ, et al. Management of large vestibular schwannoma. Part I. Planned subtotal resection followed by Gamma Knife surgery: radiological and clinical aspects. J Neurosurg 2011;115(05):875-884
15 Colotta F, Allavena P, Sica A, Garlanda C, Mantovani A. Cancerrelated inflammation, the seventh hallmark of cancer: links to genetic instability. Carcinogenesis 2009;30(07):1073-1081

16 Allavena P, Sica A, Garlanda C, Mantovani A. The Yin-Yang of tumor-associated macrophages in neoplastic progression and immune surveillance. Immunol Rev 2008;222:155-161

17 Allen M, Louise Jones J. Jekyll and Hyde: the role of the microenvironment on the progression of cancer. J Pathol 2011;223(02): 162-176

18 de Vries M, Briaire-de Bruijn I, Malessy MJ, de Bruïne SF, van der Mey AG, Hogendoorn PC. Tumor-associated macrophages are related to volumetric growth of vestibular schwannomas. Otol Neurotol 2013;34(02):347-352

19 Martinez FO, Helming L, Gordon S. Alternative activation of macrophages: an immunologic functional perspective. Annu Rev Immunol 2009;27:451-483

20 Solinas G, Germano G, Mantovani A, Allavena P. Tumor-associated macrophages (TAM) as major players of the cancer-related inflammation. J Leukoc Biol 2009;86(05):1065-1073

21 de Vries M, Hogendoorn PC, Briaire-de Bruyn I, Malessy MJ, van der Mey AG. Intratumoral hemorrhage, vessel density, and the inflammatory reaction contribute to volume increase of sporadic vestibular schwannomas. Virchows Arch 2012;460 (06):629-636

22 Monsell EM. New and revised reporting guidelines from the Committee on Hearing and Equilibrium. American Academy of Otolaryngology-Head and Neck Surgery Foundation, Inc. Otolaryngol Head Neck Surg 1995;113(03):176-178

23 House JW, Brackmann DE. Facial nerve grading system. Otolaryngol Head Neck Surg 1985;93(02):146-147

24 Gomez-Brouchet A, Delisle MB, Cognard C, et al. Vestibular schwannomas: correlations between magnetic resonance imaging and histopathologic appearance. Otol Neurotol 2001;22(01): 79-86

25 Niemczyk K, Vaneecloo FM, Lecomte MH, et al. Correlation between Ki-67 index and some clinical aspects of acoustic neuromas (vestibular schwannomas). Otolaryngol Head Neck Surg 2000;123(06):779-783

26 Lee DJ, Westra WH, Staecker H, Long D, Niparko JK, Slattery WH III. Clinical and histopathologic features of recurrent vestibular schwannoma (acoustic neuroma) after stereotactic radiosurgery. Otol Neurotol 2003;24(04):650-660, discussion 660

27 Pollock BE. Management of vestibular schwannomas that enlarge after stereotactic radiosurgery: treatment recommendations based on a 15 year experience. Neurosurgery 2006;58(02):241-248 , discussion 241-248

28 Coward J, Kulbe H, Chakravarty $\mathrm{P}$, et al. Interleukin-6 as a therapeutic target in human ovarian cancer. Clin Cancer Res 2011;17(18):6083-6096

29 Hagemann T, Lawrence T, McNeish I, et al. "Re-educating” tumorassociated macrophages by targeting NF-kappaB. J Exp Med 2008; 205(06):1261-1268

30 Pander J, Heusinkveld M, van der Straaten T, et al. Activation of tumor-promoting type 2 macrophages by EGFR-targeting antibody cetuximab. Clin Cancer Res 2011;17(17):5668-5673

31 Kandathil CK, Dilwali S, Wu C-C, et al. Aspirin intake correlates with halted growth of sporadic vestibular schwannoma in vivo. Otol Neurotol 2014;35(02):353-357

32 Kandathil CK, Cunnane ME, McKenna MJ, Curtin HD, Stankovic KM. Correlation between aspirin intake and reduced growth of human vestibular schwannoma: volumetric analysis. Otol Neurotol 2016;37(09):1428-1434

33 Dilwali S, Kao SY, Fujita T, Landegger LD, Stankovic KM. Nonsteroidal anti-inflammatory medications are cytostatic against human vestibular schwannomas. Transl Res 2015;166(01):1-11 